\section{AN OPERATION FOR THE RADICAL CURE OF HERNIA.}

By J. HOGARTH PRINGLE, M.B., F.R.C.S., Surgeon, Glasgow Royal Iufirmary.

In this paper I desire to describe a method of performing a radical operation for the cure of inguinal hernia which $I$ have now been employing for nearly eighteen months, and which has so far given results that are quite satisfactory.

Previous to that time it had been my practice to operate upon all cases according to the method of Sir William Macewen, for I well know what admirable cures are obtained by him. Formerly I had many opportunities of seeing and examining cases, often at long periods after operation at his hands, and I never saw a patient who had a recurrence of his hernia.

With the results in my cases I was, however, disappointed. Seventy-six patients were operated on by me, and of these 14 have not been seen again since very shortly after their dismissal ; possibly some of these may have had a relapse; but, of the remainder, 5 are known to me to have had a recurrence of the hernia, while in the others who have been seen from time to time since their operation the result has been good, and the inguinal canal seems to be closed perfectly. Of the 5 patients in whom a recurrence occurred, 2 had a stitch abscess after operation ; one of these went eighteen months, the other two years before recurrence took place; while of the 3 others, all of whom healed perfectly a septically, 2 relapsed within a few weeks after dismissal, the other not for eighteen months.

In some of my cases trouble was caused after operation by thrombosis of the spermatic veins, which lead to great oedema of the scrotum and the formation of a sausage-like swelling, which often extended for the whole length of the inguinal canal, where it might possibly act as a sort of wedge and tend ultimately to interfere with the accurate apposition of the walls of the canal.

It had sometimes appeared to me at the end of an operation that an unnecessarily large amount of tissue, composed of the cremasteric and other fasciae, was left to be transmitted through the inguinal canal as it had just been restored, and that this might prevent the closure of that passage being as perfect as it might be if they were not there. For this reason I frequently removed considerable portions of these tissues, and thought that a more perfect closure was obtained in consequence; but in doing this it was constantly observed that the fasciae so cut away were of considerable thickness, the cremaster itself even in cases of large herniae being often quite a good layer, and it was thought they might be advantageously utilized for the purpose of aiding to close the canal instead of removing them.

Operation.

With this idea an operation came to be performed of which the following is a description:

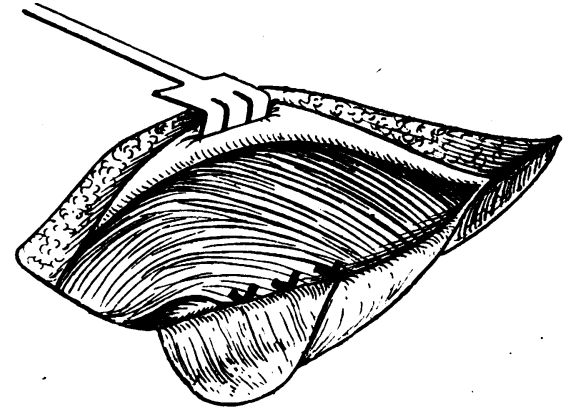

Fig $x$.

1. An incision $\frac{3}{4}$ in. above and parallel to Poupart's ligament, and running from a point over the internal pillar of the external abdominal ring to a point situated over the exteral iliac artery, is made; the external oblique is shown for the whole length of the wound and the external ring clearly defined. Tne aponeurosis of the oblique is now divided from the point of meeting of the two pillars of the ring, outwards as far as the limit of the skin wound, and its margins retracted, the lower flap being dissected off the internal oblique and cremaster down to Poupart's ligament (Fig. 1).
2. The cremaster and all tissues beneath it down to the sac are divided in a direction corresponding to the dotted line in Fig. I, and the flap, as outlined, is turned down off the sac by. blunt dissection and held back.

3. The sac is now stripped carefully off the cord right up to the internal abdominal ring, In cases where the sac is sufficiently thick to permit the necessary manipulation it is returned in the following way: A ligature is tied round the fundus, or, if any of the sac has been cut away, round the most distal portion of what is leit, and one end of the ligature, which is left long, is used as a continuous suture, which passes from side to side, and, in a sense, spirally, up the sac, which is at the same time continually invaginated. The result is that a"balling" of the sac is produced, and in this way a thick and rounded pad is formed, which comes to lie. exactly at the internal abdominal ring, and to project into the peritoneal cavity as a slightly rounded prominence. If the hernia is recent and the sac thin, it is very troublesome to get it to invaginate satisfactorily; it is then returned by the running suture, as recommended by Sir William Macewen.

4. The distal end of the cord is now drawn upon from the wound ; the spermatic veins isolated, and divided between two ligatures just proximal to the epididymis: the ligatures are then tied together so as to keep the venous stumps approximated in order that some traction may be exerted upon the cord at a later stage.

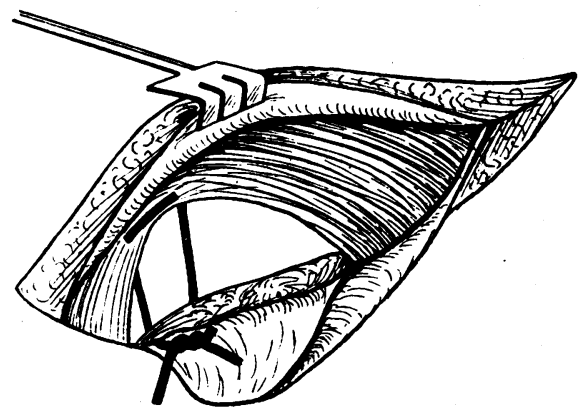

Fig. 2.

5uture of the Internal Oblique.-A " relaxing" suture-of catgut of medium strength - threaded on two ntedles is next passed through the horizontal portion of the combined tendons of the transversalis and internal oblique, just as they approach the rectus muscle, at two points from without inwards so as to leave a loop of the suture lying upon the outer aspect of the tendon: the two needles are then passed behind the rord and through Poupart's ligament (as in Fig. 2); the needle which is placed nearer the middle line being passed close up to the spine of the pubes, the other at the point where the s permatic cord naturally crosses the ligament. This suture is meantime left untied. Several catgut sutures are then passed between the internal oblique and Poupart's ligament co as to get as close coaptation of these two structures as possible from the insertion of Poupart at the pubes to the point where it is crossed by the cord, these sutures all being

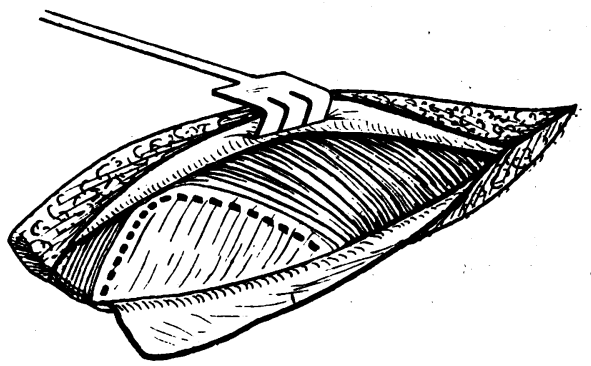

Fig. 3 .

posterior to the latter. It often happens that the sheath of the rectus (here not a "sheath" at all in the sense of there being two layers, but actually the combined tendons of the transversalis and oblique passing in front of the rectus) is too rigid to draw down easily. In these cases its attachments to the anterior surface of the tendon of the rectus are freely divided, which always permits it being easily approximated to Poupart; and if, as is sometim ${ }^{\circ}$ s the case, the rectus sends 
a prolongation outwards to be inserted into the ileo-pectineal line, this is also divided close to the bone and sutured to Poupart, and thereby much additional strength is given to the inner part of the posterior wall of the canal. In tying these sutures, the "relaxing" suture is first tied and the others after it.

An assistant now draws upon the testicle so as to stretch the cord, and three or four sutures are passed external to the latter between the oblique and Poupart so as to draw the muscle down in front of the cord (see Fig. 3).

6. The cremaster flap is drawn upwards and inwards as far as possible, and sutured to the anterior surface of the internal oblique and of its tendon over the rectus by deep as well as marginal sutures. (The appearance of the wound at this stage is shown in Fig. 4.)

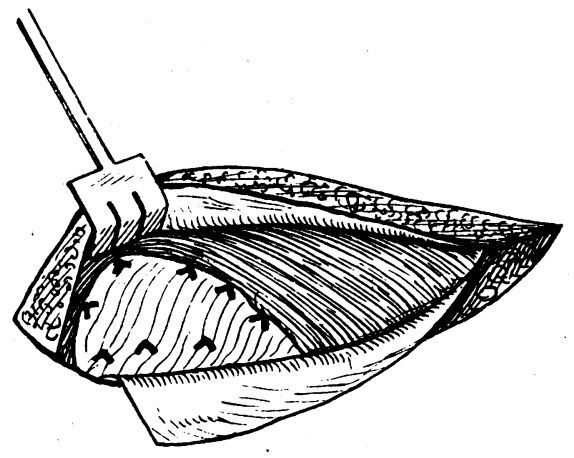

Fig. 4 .

7. The aponeurosis of the external oblique is now sutured as close as possible; a buried suture is used for the subcutaneous tissue layer, and finally the skin is sutured.

RESULTS.

Twenty patients have now been operated upon by me in this manner, and although no great interval has elapsed since the first case was so treated, there is no instance of any recurrence of the hernia.

All the cases have been seen frequently, and the region of the operation in every one is firm and tight.

None of the patients after operation developed any thickening of the cord above the level at which the veins were ligatured; and the object for which that procedure was adopted appears, therefore, to have been attained. Oedema of the scrotum has been very markedly less than in my former experience, and only one of the patients developed a hydrocele.

I believe strongly in the advantage of a pad formed from the hernial sac as advocated by sir William Macewen, and think that the method I employ for making it is a good one. in a patient recently operated upon who had both an irreducible inguinal and umbilical hernia, the inguinal was treated in the way above described in the first place, and when the umbilical hernia was dealt with I took the opportunity of examining the state of the inguinal region from the abdominal aspect, and found the closure to be as accurate as it could possibly be The peritoneal pad was tight and hard and rounded, projecting above the surface of the abdominal wall at the internal ring like one-half of a small ball.

There is no transplantation of the cord which forms such a prominent feature of the Bassini-Halsted operation. I take every care that the cord shall cross Poupart's ligament at its normal situation. The cord, however, in my operation is practically reduced to the vas and its own vessels, for by tying and dividing the veins I get rid of them as venous channels as completely as if they were actually excised; the amount of tissue they represent in their collapsed condition is very small indeed, so that the opening through which the cord has to be transmitted beneath the internal oblique is of the smallest diameter.

My reason for dealing with the veins in this way is that I believe it offers the best means of preserving intact the nerve to the cremaster. Normally this nerve runs in close proximity to the spermatic veins at the posterior and outer part of the cord; if it can be preserved the substance of the cremaster flap will thereby be preserved, and in that case will be of much greater value than it can be if it atrophy, as must happen if the nerve be destroyed. When the cremaster flap is sutured into position on the surface of the oblique it is drawn both upwards and inwards a little further than its actual natural position (compare Figs. I and 4); the two structures mutually support each other, and form a small canal in which lies the vas along with the collapsed veins. The flap remains a taut layer, always, as I have found, of a definite value, which, however, will vary in different cases. I have been struck with the degree of contraction possessed by the fibres of what at first sight looks like an attenuated and thin muscle. After the flap is cut and dissected down it will contract into quite a compact layer.

I have quite recently learned that an account of an operation somewhat similar to what has just been described was published by Halsted in the Johns Hopkins Bulletin for August, 1903: it constitutes a very marked modification of the operation which has hitherto been known by his name. In his new operation Halsted removes the sac, excises the veins, and employs the cremaster as an additional layer of tissue much in the same way as I have done; but he places it deeper than the internal oblique, suturing it to the under surface of the latter.

It appears to me to be distinctly preferable that the flap made of cremaster and the other fasciae should be placed in front of the internal oblique; it does not interfere in any way with the suturing of the oblique to Poupart's ligament, and it has, as it seems to me, this great advantage, that nothing remains except the vas-and in my operation the empty veins - to be passed beneath the inferior border of the oblique. Whereas if it be placed deeper than the oblique there will be a puckered-up portion of these fasciae to be transmitted, and consequently, a less close approximation of the oblique to Poupart is possible, unless the flap is cut away from its prolongation into the scrotum-a procedure which I do not gather from his description is carried out by Halsted.

Excision of the spermatic veins is, I believe, absolutely certain to lead to division of the cremasteric nerve and consequent atrophy of the cremaster fibres, a result which in $\mathrm{my}$ method I have all along done my utmost to avoid, and as I believe with success, for several of the patients have shown a good cremasteric reflex after operation.

\section{Femorac Hernia.}

In my operations for this variety of hernia after freeing the sac I return it in the same manner as above described so as to invaginate and "ball" it. Then I make a longitudinal incision at the inner part of the femoral sheath, so as to open the venous or middle compartment, free the inner border of the femoral vein with the handle of the knife and draw the vein outwards. Then pass a needle carrying a strong catgut suture from the venous compartment (and therefore to the outer side of the septum between it and the "femoral canal") through the pectineus muscle, and bring it out close to Gimbernat's ligament; the two ends of the suture are then passed from below upwards through Poupart, and, at the inner side, through Gimbernat.

This suture lies entirely outside the walls of the femoral canal, and when it is pulled up and tied closes the latter completely; no chink is left between the margin of the ring and one of the sutures through which a hernia can wedge its way.

Further closure of the canal and reinforcement of this deep suture are obtained by two or three interrupted stitches which are passed between Poupart and the pubic portion of the fascia lata over the pectineus, and the wound is closed by suturing the subcutaneous tissues and then the skin.

No case which I have operated upon by this method is known to me to have had a recurrence of the hernia.

ON GASTRO-JEJUNOSTOMY FOR PYLORIC

\section{STENOSIS, GASTRIC ULCER AND SOME OTHER} NON-MALIGNANT CONDITIONS.

By GILBERT BARLING, M.B., F.R.C.S.,

Professor of Surgery University of Birmingham, Surgeon, Birmingham General Hospital.

Mx observations will not be rigidly circumscribed to the title of my paper as there are operations similar in purpose to gastro-jejunostomy to which I must briefly refer, so also some other conditions than actual pyloric stenosis and existing gastric ulcer will be considered, as duodenal ulcer, for instance, and hour-glass stomach. The radical procedure of excision of the pylorus or some other area of the stomach I have, however, purposely excluded as these would expand my remarks to undue length. In my own hands 'gastro-jejunostomy has 